\title{
THE CONVERSE TO THE FIXED POINT THEOREM OF P. A. SMITH ${ }^{1}$
}

\author{
BY LOWELL JONES
}

Communicated by P. Emery Thomas, September 22, 1971

$\boldsymbol{Z}_{n}, Z_{n}$ will denote the multiplicative, and additive cyclic groups of order $n$.

Recall that a simplicial complex $K$ is a $Z_{n}$-homology manifold if the link of every simplex in $K$ is a $Z_{n}$-homology sphere. A $Z_{n}$-homology manifold pair $(K, \partial K)$ is defined similarly. A group action $G \times X \rightarrow X$ on the space $X$ is semifree if, for each $x \in X, G$ acts either trivially or freely on the orbit of $x$.

Let $n$ be an even integer, and $(K, \partial K) \subset\left(D^{m}, \partial D^{m}\right)$ a combinatorial embedding having even codimension $\geqq 6$ and further satisfying $K \cap \partial D^{m}=\partial K$.

THEOREM. $K \subset D^{m}$ is the fixed point set of a semifree, combinatorial group action $Z_{n} \times D^{m} \rightarrow D^{m}$ if and only if

(1) $\bar{H}\left(K, Z_{n}\right)=0$,

(2) $(K, \partial K)$ is a $Z_{n}$-homology manifold pair.

The "only if" part of the theorem was proven by P. A. Smith [3].

The theorem holds for $n$ odd provided the regular neighborhood for $(K, \partial K)$ in $\left(D^{m}, \partial D^{m}\right)$ admits a one-parameter cross section, e.g., $(K, \partial K)$ $\subset\left(D^{m}, \partial D^{m}\right)$ factors as $(K, \partial K) \subset\left(D^{m-2}, \partial D^{m-2}\right) \subset\left(D^{m}, \partial D^{m}\right)$.

A classification theorem can also be proven, which gives a bijective correspondence between equivalence classes of semifree $\boldsymbol{Z}_{n}$-actions on $D^{m}$ having $K \subset D^{m}$ for fixed point set and the elements of $\boldsymbol{H}_{0}(K, \partial K)$, where $\boldsymbol{H}_{*}(\mathrm{)})$ is a certain computable homology functor.

These results extend to the following situations:

(1) when $M$ replaces $D^{m}$, where $M$ is a simply connected manifold satisfying $\bar{H}\left(M, Z_{n}\right)=0$,

(2) a relative version of (1),

(3) replacing $Z_{n}$ by any finite group which acts freely on the sphere normal to $K$ in $D^{m}$.

In order to prove the above results the author has been led to

(a) the extension of the Characteristic Variety Theorem [4] to the nonsimply connected case (see [1]), and

(b) the extension of transversality and surgery techniques to the Poincaré duality category (see [2]).

AMS 1970 subject classifications. Primary 57C99.

${ }^{1}$ Partially supported by NSF GP-22723 and NSF GP-29073. 


\section{BIBLIOGRAPHY}

1. L. E. Jones, Combinatorial symmetries of the m-disc, Lecture Notes, Berkeley, Calif., 1971.

2. —_ Patch spaces, Lecture Notes, Berkeley, Calif., 1971.

3. P. A. Smith, "Fixed points of periodic transformations," Appendix B in S. Lefschetz, Algebraic topology, Amer. Math. Soc. Colloq. Publ., vol. 27, Amer. Math. Soc., Providence, R.I., 1942, pp. 350-373. MR 4, 84.

4. D. Sullivan, Triangulating and smoothing homotopy equivalences and homeomorphisms, Lecture Notes, Princeton University, Princeton, N.J., 1967.

5. C. T. C. Wall, Surgery on compact manifolds, London Math. Soc. Monographs, no. 1, Academic Press, New York, 1970.

Department of Mathematics, University of California, Berkeley, California 94720 